\title{
Checklist dos Copepoda Cyclopoida de vida livre de água doce do Estado de São Paulo, Brasil
}

\author{
William Marcos da Silva ${ }^{2}$ \& Takako Matsumura-Tundis ${ }^{1,3}$ \\ ${ }^{1}$ Instituto Internacional de Ecologia, Rua Bento Carlos, 750, CEP: 13560-660, São Carlos, SP, Brasil \\ ${ }^{2}$ Universidade Federal do Mato Grosso do Sul - UFMS, Campus do Pantanal, \\ e-mail:wmsilvax@gmail.com \\ ${ }^{3}$ Autor para correspondência: Takako Matsumura-Tundisi,e-mail: takako@iie.com.br
}

SILVA, W.M. \& MATSUMURA-TUNDISI, T. Checklist of fresh-water free living Copepoda Cyclopoida from São Paulo State, Brazil. Biota Neotrop. 11(1a): http://www.biotaneotropica.org.br/v11n1a/en/abstract?i nventory+bn0261101a2011.

Abstract: The biodiversity of Cyclopoida copepods of São Paulo State was studied during the Program BIOTA/ FAPESP. Samples were collected from 207 water bodies of the 22 Units of Management of Water Resources (UGRHI). From the 39 registered species, 6 were new records from São Paulo State. The new species Thermocyclops iguapensis is a species restricted to the Atlantic coast (Silva \& Matsumura-Tundisi 2005a). For some species corrections of identification were made: species identified as Mesocyclops kieferi, Mesocyclops brasilianus, and Paracyclops fimbriatus are in reality respectively Mesocyclops ogunnus, Mesocyclops meridianus and Paracyclops chiltoni. These corrections are important since their populations can be indicators of the trophic conditions as verified by Silva \& Matsumura-Tundisi (2002) for the reservoirs of the lower and middle Tietê River, São Paulo State.

Keywords: fresh-water Copepoda Cyclopoida, biodiversity of the State of São Paulo, BIOTA/FAPESP Program.

Number of species: in the world: 609, in Brazil: 84, estimated in São Paulo State: 39.

SILVA, W.M. \& MATSUMURA-TUNDISI, T. Checklist dos Copepoda Cyclopoida de vida livre de água doce do Estado de São Paulo, Brasil. Biota Neotrop. 11(1a): http://www.biotaneotropica.org.br/v11n1a/pt/ab stract?inventory+bn0261101a2011.

Resumo: Estudo da biodiversidade de Copepoda Cyclopoida do Estado de São Paulo foi feito no Programa BIOTA/ FAPESP, amostrando 207 corpos de água das 22 Unidade de Gerenciamento de Recursos Hídricos (UGRHI). Foram registradas 39 espécies englobando tanto espécies de hábito planctônico como de hábito não planctônico. Destas, 6 espécies constituem registro novo no Estado de São Paulo sendo uma espécie nova, o Thermocyclops iguapensis, espécie restrita na costa Atlântica (Silva \& Matsumura-Tundisi 2005a). Foram realizadas também correções de erros de identificação para algumas espécies: as espécies anteriormente identificadas como Mesocyclops kieferi, Mesocyclops brasilianus, e Paracyclops fimbriatus correspondem respectivamente Mesocyclops ogunnus, Mesocyclops meridianus e Paracyclops chiltoni. Estas correções são importantes, pois a correta identificação de uma espécie que pode vir formar a população mais significativa de um ambiente pode servir de indicativo das condições tróficas como verificado por Silva \& Matsumura-Tundisi (2002) para relações entre espécies de Cyclopoida e o grau de trofia de reservatórios do médio e baixo Tietê.

Palavras-chave: Copepoda Cyclopoida de água doce, biota paulista, Programa BIOTA/FAPESP.

Número de espécies: no mundo: 609, no Brasil: 84, estimadas no Estado de São Paulo: 39. 


\section{Introdução}

Os Cyclopoida são os copépodos mais abundantes e de maior sucesso nos sistemas dulciaquícolas, podendo ser encontrados em rios, riachos, lagos, represas, áreas alagadas e corpos de água temporários (Huys \& Boxshall, 1991). Estudos para o conhecimento da biodiversidade dos Cyclopoida, assim como os de outros grupos, têm sido realizados de maneira fragmentada e concentrados em certas localidades. Em revisão das espécies de Copepoda Cyclopoida neotropicais, Silva (2008) verificou que a riqueza de espécies e endemismo das localidades está diretamente ligada ao número de pesquisadores da região.

Um fato importante na taxonomia de Copepoda Cyclopoida atual, foi a mudança de paradigma sobre o cosmopolitismo das espécies. Revisões realizadas sobre o grupo vêm mostrando que as espécies restritas geograficamente são em maior número que as de ampla distribuição (Silva, 2008). Este fato aumenta a importância de estudos taxonômicos, pois estas espécies podem refletir as alterações ambientais e mostrar limites tanto para proteção quanto para intervenção, em áreas de importância ecológica e econômica.

\section{Metodologia}

Para a elaboração da lista de Copepoda Cyclopoida de vida livre do Estado de São Paulo foram consultadas as seguintes referências: Arcifa
(1984), Claus (1893), Dussart \& Defaye (1985), Dussart (1984, 1987), Gutierrez-Aguirre \& Suarez-Morales (2001), Karaytug (1999), Kiefer (1936, 1956, 1927, 1929, 1925, 1931, 1933, 1976, 1981), Lowndes (1934), Matsumura-Tundisi \& Silva (1999, 2002), Matsumura-Tundisi \& Rocha (1983), Matsumura-Tundisi et al. (1990), Nogueira (2002), Nogueira et al. (2004), Reid (1989, 1985), Rocha \& Bjornberg (1987), Rocha \& Botelho (1998), Rocha (1999), Rocha et al. (1995), Sars (1901), Sendacz \& Kubo (1982), Sendacz (1993), Silva \& MatsumuraTundisi (2002, 2005a), Silva (2003), Tundisi (1980).

\section{Resultados \& Discussão}

\section{Comentário sobre a lista}

A Tabela 1 mostra a lista de espécies de Copepoda Cyclopoida de água doce do Estado de São Paulo. A lista apresenta 39 espécies distribuídas em 14 gêneros. No mundo são 609 espécies distribuídas em 43 gêneros. Na região neotropical são 148 espécies distribuídas em 22 gêneros e no Brasil são 84 espécies em 22 gêneros. A Tabela 2 mostra os corpos de água amostrados nas 22 UGRHI do Estado de São Paulo com as respectivas coordenadas. A Figura 1 refere-se ao mapa do Estado de São Paulo com as 22 UGRHI.

As espécies registradas nos corpos de água dos Estados do Amazonas, Pará e Distrito Federal (Rocha \& Botelho 1998), são

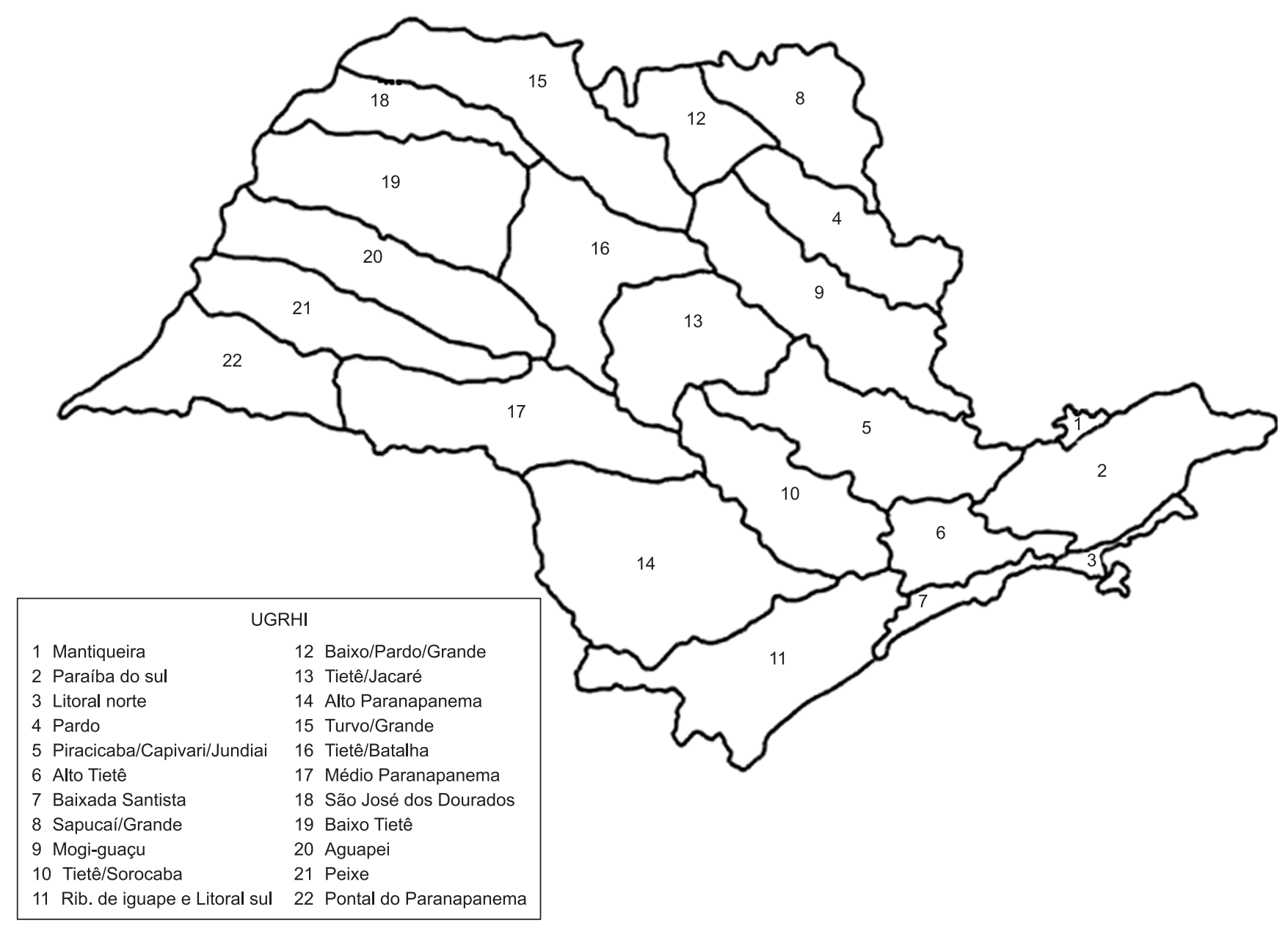

Figura 1. Estado de São Paulo e suas 22 unidades hidrográficas de gerenciamento.

Figure 1. São Paulo State and its 22 hydrographic management units. Portugues: 


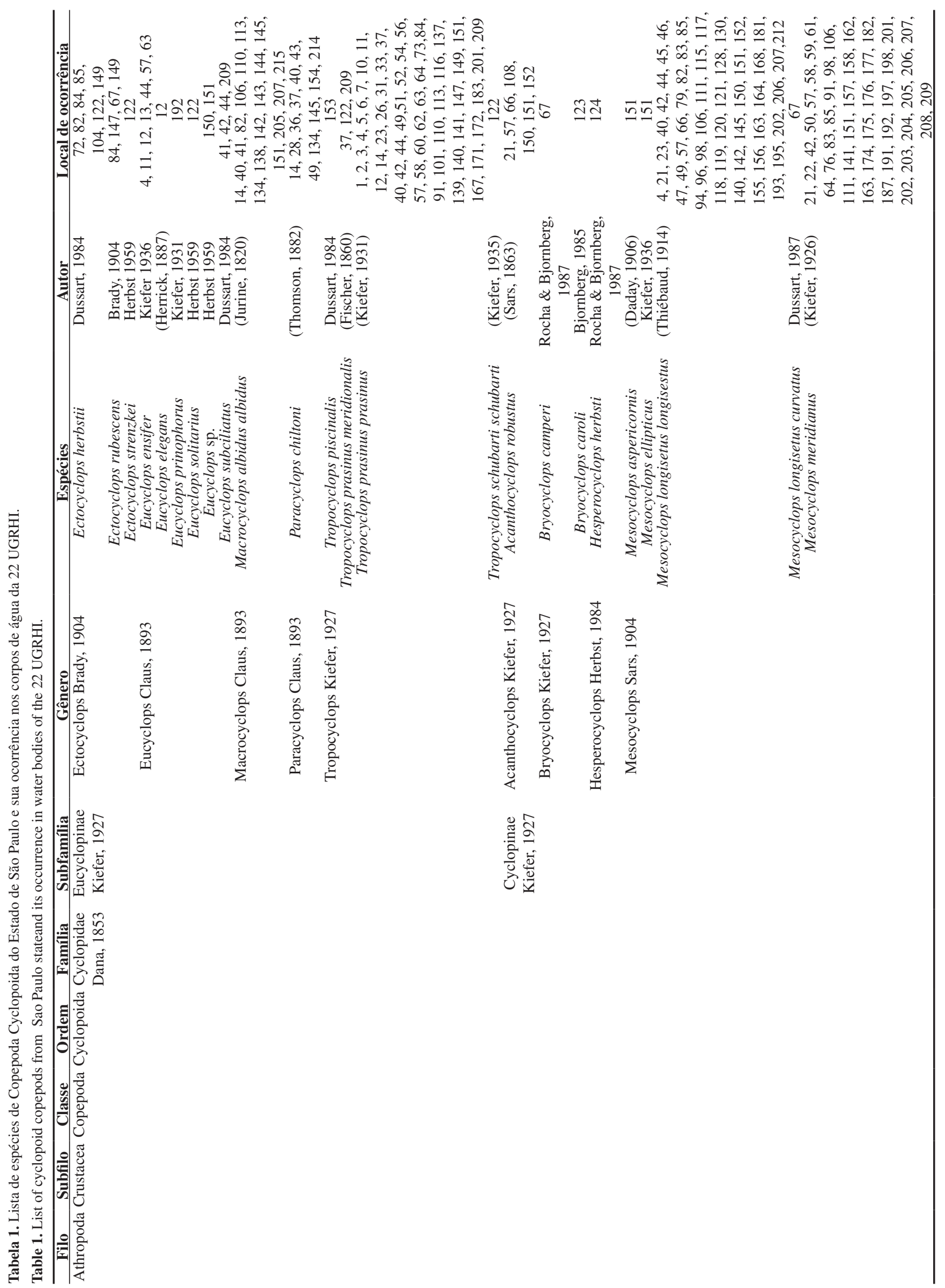


Silva, W.M. \& Matsumura-Tundisi, T.

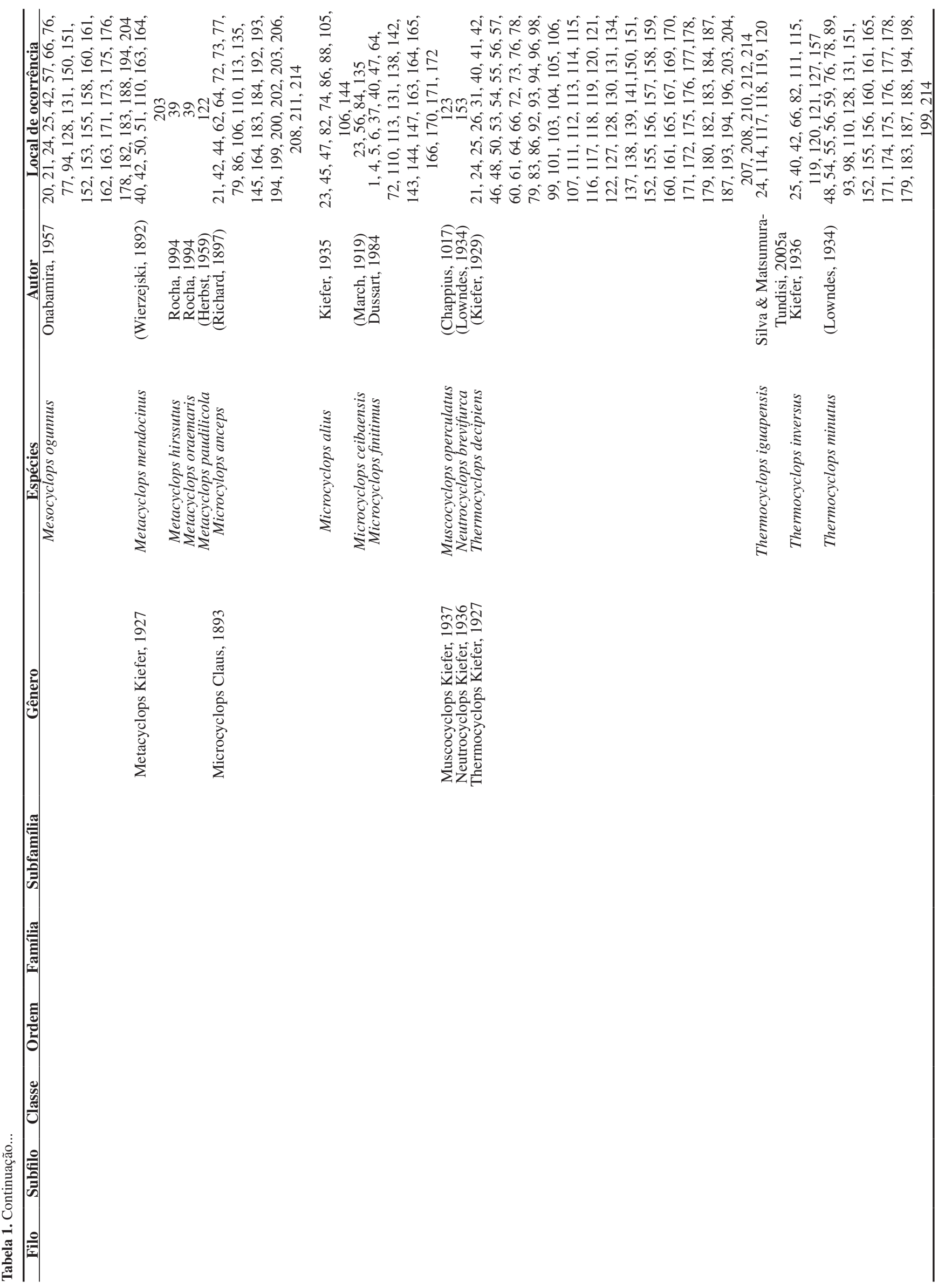


Tabela 2. Lista dos corpos de água amostrados por UGRHI e suas receptivas coordenadas geográficas em Latitude e Longitude. Os corpos de água foram assim classificados: Hidrelétricas (He), Represas (Re) (abastecimento, piscicultura, irrigação e desedentação de animais), Redutores de velocidade (RV) (usados para abastecimento), Lagoa Marginal (LM), (Lagoa, área inundada) e Rio.

Table 2. List of water bodies sampled by UGRHI and its respective coordinates. The Water bodies Classification: Hydroelectric power plan (He) (Water supply,pisciculture, irrigation, watering breeding),Water speed reducer (RV)(water supply), Marginal lakes (LM)(Ponds, floodplain areas) and River (Rio).

\begin{tabular}{|c|c|c|c|}
\hline & Corpos de água & Latitude & Longitude \\
\hline \multicolumn{4}{|c|}{ UHGRH 1 Mantiqueira } \\
\hline 1 & Re Fojo Est. I & $22^{\circ} 42.910^{\prime} \mathrm{S}$ & $045^{\circ} 32.097^{\prime} \mathrm{W}$ \\
\hline 2 & LM Fojo 1 & $22^{\circ} 42.946^{\prime} \mathrm{S}$ & $045^{\circ} 32.087^{\prime} \mathrm{W}$ \\
\hline 3 & LM Lambaris & $22^{\circ} 41.394^{\prime} \mathrm{S}$ & $045^{\circ} 28.966^{\prime} \mathrm{W}$ \\
\hline 4 & LM Ninfóides & $22^{\circ} 41.442^{\prime} \mathrm{S}$ & $045^{\circ} 29.140^{\prime} \mathrm{W}$ \\
\hline 5 & Re H. Florestal . 1 & $22^{\circ} 41.43^{\prime} \mathrm{S}$ & $045^{\circ} 29.140^{\prime} \mathrm{W}$ \\
\hline 6 & Re H. Florestal -. 2 & $22^{\circ} 41.422^{\prime} \mathrm{S}$ & $045^{\circ} 29.140^{\prime} \mathrm{W}$ \\
\hline 7 & Re H. Florestal -. 3 & $22^{\circ} 41.40^{\prime} \mathrm{S}$ & $045^{\circ} 29.140^{\prime} \mathrm{W}$ \\
\hline 8 & Re H. Florestal .4 & $22^{\circ} 41.392^{\prime} \mathrm{S}$ & $045^{\circ} 29.140^{\prime} \mathrm{W}$ \\
\hline 9 & Re H. Florestal . 5 & $22^{\circ} 41.3^{\prime} \mathrm{S}$ & $045^{\circ} 29.140^{\prime} \mathrm{W}$ \\
\hline 10 & Re Sta Isabel Est.I & $22^{\circ} 43.582^{\prime} \mathrm{S}$ & $045^{\circ} 27.016^{\prime} \mathrm{W}$ \\
\hline 11 & Rio das Trutas & $22^{\circ} 43.343^{\prime} \mathrm{S}$ & $045^{\circ} 27.096^{\prime} \mathrm{W}$ \\
\hline 12 & Re Tundra & $22^{\circ} 43.304^{\prime} \mathrm{S}$ & $045^{\circ} 27.130^{\prime} \mathrm{W}$ \\
\hline 13 & Re Lavrinhas 1 & $22^{\circ} 42.130^{\prime} \mathrm{S}$ & $045^{\circ} 25.202^{\prime} \mathrm{W}$ \\
\hline 14 & Re Lavrinhas 2 & $22^{\circ} 41.846^{\prime} \mathrm{S}$ & $045^{\circ} 25.154^{\prime} \mathrm{W}$ \\
\hline 15 & Re Inst. Pesca & $22^{\circ} 42.846^{\prime} \mathrm{S}$ & $045^{\circ} 28.154^{\prime} \mathrm{W}$ \\
\hline 16 & Re Itapeva - pelág. & $22^{\circ} 46.190^{\prime} \mathrm{S}$ & $045^{\circ} 31.794^{\prime} \mathrm{W}$ \\
\hline 17 & Re Hípica 1 & $22^{\circ} 43.394^{\prime} \mathrm{S}$ & $045^{\circ} 33.093^{\prime} \mathrm{W}$ \\
\hline 18 & Re Hípica 2 & $22^{\circ} 43.346^{\prime} \mathrm{S}$ & $045^{\circ} 33.070^{\prime} \mathrm{W}$ \\
\hline 19 & Re Vila Inglesa & $22^{\circ} 44.474^{\prime} \mathrm{S}$ & $045^{\circ} 34.106^{\prime} \mathrm{W}$ \\
\hline \multicolumn{4}{|c|}{ UHGRH 2- Paraiba do Sul } \\
\hline 20 & He Santa Branca & $23^{\circ} 22^{\prime} 05,5^{\prime \prime} \mathrm{S}$ & $045^{\circ} 51^{\prime} 28,2^{\prime \prime} \mathrm{W}$ \\
\hline 21 & He Paraibuna & $23^{\circ} 26^{\prime} 53,2^{\prime \prime} \mathrm{S}$ & $045^{\circ} 33^{\prime} 43,5^{\prime \prime} \mathrm{W}$ \\
\hline 22 & LM Olaria & $22^{\circ} 49^{\prime} 06,5^{\prime \prime} \mathrm{S}$ & $045^{\circ} 13^{\prime} 24,2^{\prime \prime} \mathrm{W}$ \\
\hline 23 & LM Marginal & $23^{\circ} 49^{\prime} 19,1$ " S & $045^{\circ} 12^{\prime} 45,3^{\prime \prime} \mathrm{W}$ \\
\hline 24 & He Funil & $22^{\circ} 30^{\prime} 43,3 ” \mathrm{~S}$ & $044^{\circ} 38^{\prime} 01,2^{\prime \prime} \mathrm{W}$ \\
\hline 25 & Re Hotel & $22^{\circ} 37^{\prime} 32,5^{\prime \prime} \mathrm{S}$ & $044^{\circ} 38^{\prime} 47,7^{\prime \prime} \mathrm{W}$ \\
\hline 26 & Rio Parque Bocaina & $22^{\circ} 44^{\prime} 33^{\prime \prime} \mathrm{S}$ & $044^{\circ} 36^{\prime} 58,2^{\prime \prime} \mathrm{W}$ \\
\hline 27 & Re Ch. S.Bocaina & $22^{\circ} 38^{\prime} 54,4 " \mathrm{~S}$ & $044^{\circ} 35^{\prime} 37,6^{\prime \prime} \mathrm{W}$ \\
\hline \multicolumn{4}{|c|}{ UHGRH 3- Litoral Norte } \\
\hline 28 & Re Rio Macaco & $23^{\circ} 25^{\prime} 29,9^{\prime \prime} \mathrm{S}$ & $45^{\circ} 08^{\prime} 09,5^{\prime \prime} \mathrm{W}$ \\
\hline 29 & RV Rio Grande & $23^{\circ} 23^{\prime} 42,8^{\prime \prime} \mathrm{S}$ & $45^{\circ} 07^{\prime} 10^{\prime \prime} \mathrm{W}$ \\
\hline 30 & RV Rio Piaba & $23^{\circ} 31^{\prime} 45,0^{\prime \prime} \mathrm{S}$ & $45^{\circ} 15^{\prime} 28,1^{\prime \prime} \mathrm{W}$ \\
\hline 31 & RV Cantinho do Céu & $23^{\circ} 31^{\prime} 54,1^{\prime \prime} \mathrm{S}$ & $45^{\circ} 15^{\prime} 31,5^{\prime \prime} \mathrm{W}$ \\
\hline 32 & L Poção & $23^{\circ} 35^{\prime} 40,7^{\prime \prime} \mathrm{S}$ & $45^{\circ} 23^{\prime} 35,5^{\prime \prime} \mathrm{W}$ \\
\hline 33 & Rio Guaximduba & $23^{\circ} 36^{\prime} 29,3^{\prime \prime} \mathrm{S}$ & $45^{\circ} 23^{\prime} 39,4^{\prime \prime} \mathrm{W}$ \\
\hline 34 & Rio Baixo & $23^{\circ} 41^{\prime} 55,8^{\prime \prime} \mathrm{S}$ & $45^{\circ} 29^{\prime} 14,0^{\prime \prime} \mathrm{W}$ \\
\hline 35 & RV Rio Macaco & $23^{\circ} 32^{\prime} 08,0^{\prime \prime} \mathrm{S}$ & $45^{\circ} 17^{\prime} 30,6^{\prime \prime} \mathrm{W}$ \\
\hline 36 & RV S.Francisco & $23^{\circ} 45^{\prime} 28,8^{\prime \prime} \mathrm{S}$ & $45^{\circ} 25^{\prime} 14,3^{\prime \prime} \mathrm{W}$ \\
\hline 37 & RV Água Branca & $23^{\circ} 50^{\prime} 11,7^{\prime \prime} \mathrm{S}$ & $45^{\circ} 21^{\prime} 30,3^{\prime \prime} \mathrm{W}$ \\
\hline 38 & RV Pombo II & $23^{\circ} 52^{\prime} 04,4^{\prime \prime} \mathrm{S}$ & $45^{\circ} 25^{\prime} 29,2$ " W \\
\hline \multicolumn{4}{|c|}{ UHGRH 4- Pardo } \\
\hline 39 & Re Graminha & $21^{\circ} 34 .^{\prime} 48,2^{\prime \prime} \mathrm{S}$ & $47^{\circ} 37^{\prime} 9,6 \mathrm{~W}$ \\
\hline 40 & Re Itaiquara & $21^{\circ} 35^{\prime} 4,98^{\prime \prime} \mathrm{S}$ & $46^{\circ} 44^{\prime} 52^{\prime} \mathrm{W}$ \\
\hline 41 & Re Faz Graminha & $21^{\circ} 32^{\prime} 55,8^{\prime \prime} \mathrm{S}$ & $46^{\circ} 49^{\prime} 36,2^{\prime \prime} \mathrm{W}$ \\
\hline 42 & He Euclides da Cunha & $21^{\circ} 36^{\prime} 3,1^{\prime \prime} \mathrm{S}$ & $46^{\circ} 56^{\prime} 54,4^{\prime \prime} \mathrm{W}$ \\
\hline 43 & Re FazSanta Helena & $21^{\circ} 32^{\prime} 06^{\prime \prime} \mathrm{S}$ & $46^{\circ} 50^{\prime} 29,7$ ' W \\
\hline
\end{tabular}


Tabela 2. Continuação...

\begin{tabular}{|c|c|c|c|}
\hline & Corpos de água & Latitude & Longitude \\
\hline \multicolumn{4}{|c|}{ UHGRH 5- Piracicaba/Capivari/Jundiai } \\
\hline 44 & He Igaratá & $23^{\circ} 11^{\prime} 24,78^{\prime \prime} \mathrm{S}$ & $46^{\circ} 07^{\prime} 14,5^{\prime \prime} \mathrm{W}$ \\
\hline 45 & He Cachoeira & $23^{\circ} 07^{\prime} 30,54^{\prime \prime} \mathrm{S}$ & $46^{\circ} 17^{\prime} 18,9^{\prime \prime} \mathrm{W}$ \\
\hline 46 & Re Piracaia & $23^{\circ} 04^{\prime} 14,46^{\prime \prime} \mathrm{S}$ & $46^{\circ} 19^{\prime} 19,4^{\prime \prime} \mathrm{W}$ \\
\hline 47 & Re Atibainha & $23^{\circ} 10^{\prime} 33,3 " \mathrm{~S}$ & $46^{\circ} 23^{\prime} 12,2^{\prime \prime} \mathrm{W}$ \\
\hline 48 & Re Atibaia & $23^{\circ} 05^{\prime} 29,34^{\prime \prime} \mathrm{S}$ & $46^{\circ} 38^{\prime} 8,16^{\prime \prime} \mathrm{W}$ \\
\hline 49 & He Salto Grande (Americana) & $22^{\circ} 42^{\prime} 19^{\prime \prime} \mathrm{S}$ & $47^{\circ} 15^{\prime} 54^{\prime \prime} \mathrm{W}$ \\
\hline \multicolumn{4}{|c|}{ UHGRH 6 - Alto Tiete } \\
\hline 50 & Re Pedro Beicht & $23^{\circ} 43.052^{\prime} \mathrm{S}$ & $046^{\circ} 57.636^{\prime} \mathrm{W}$ \\
\hline 51 & Re Cach. das Graças & $23^{\circ} 39.224^{\prime} \mathrm{S}$ & $046^{\circ} 58.062^{\prime} \mathrm{W}$ \\
\hline 52 & Re P. Paiva Castro & $23^{\circ} 19.935^{\prime} \mathrm{S}$ & $046^{\circ} 39.247^{\prime} \mathrm{W}$ \\
\hline 53 & Re. Águas Claras & $23^{\circ} 23.910^{\prime} \mathrm{S}$ & $046^{\circ} 39.524^{\prime} \mathrm{W}$ \\
\hline 54 & L.Parque Ecológico 1 & $23^{\circ} 29,196^{\prime} \mathrm{S}$ & $046^{\circ} 30,805^{\prime} \mathrm{W}$ \\
\hline 55 & L.Parque Ecológico 2 & $23^{\circ} 29.071^{\prime} \mathrm{S}$ & $046^{\circ} 31,080^{\prime} \mathrm{W}$ \\
\hline 56 & Re. Billings (Riacho Grande) & $23^{\circ} 47.488^{\prime} \mathrm{S}$ & $046^{\circ} 32,681^{\prime} \mathrm{W}$ \\
\hline 57 & Re Billings (Estoril) & $23^{\circ} 45.894^{\prime} \mathrm{S}$ & $046^{\circ} 30.966^{\prime} \mathrm{W}$ \\
\hline 58 & L. Rib. Pires & $23^{\circ} 41.318^{\prime} \mathrm{S}$ & $046^{\circ} 22.419^{\prime} \mathrm{W}$ \\
\hline 59 & Re Taiaçupeba & $23^{\circ} 34.801^{\prime} \mathrm{S}$ & $046^{\circ} 16.922^{\prime} \mathrm{W}$ \\
\hline 60 & Re Jundiaí & $23^{\circ} 39.017^{\prime} \mathrm{S}$ & $046^{\circ} 11.516^{\prime} \mathrm{W}$ \\
\hline 61 & Re Ribeirão do Campo & $23^{\circ} 38.690^{\prime} \mathrm{S}$ & $045^{\circ} 49.882^{\prime} \mathrm{W}$ \\
\hline 62 & L. Aterro & $23^{\circ} 38.742^{\prime} \mathrm{S}$ & $045^{\circ} 51.649^{\prime} \mathrm{W}$ \\
\hline 63 & Re Ponte Nova & $23^{\circ} 35.834^{\prime} \mathrm{S}$ & $045^{\circ} 56.786^{\prime} \mathrm{W}$ \\
\hline 64 & Re Pirapora & $23^{\circ} 23.479^{\prime} \mathrm{S}$ & $046^{\circ} 59.663^{\prime} \mathrm{W}$ \\
\hline 65 & Re Guarapiranga & $23^{\circ} 40^{\prime} 48,3^{\prime \prime} \mathrm{S}$ & $46^{\circ} 43^{\prime} 24,9^{\prime \prime} \mathrm{W}$ \\
\hline \multicolumn{4}{|c|}{ UHGRH 7- Baixada Santista } \\
\hline 66 & RV Sub-Alvea & $23^{\circ} 53^{\prime} 13,9^{\prime \prime} \mathrm{S}$ & $46^{\circ} 27^{\prime} 10,1^{\prime \prime} \mathrm{W}$ \\
\hline 67 & RV Jurubatuba & $23^{\circ} 51^{\prime} 12,2 ” \mathrm{~S}$ & $46^{\circ} 16^{\prime} 20,5^{\prime \prime} \mathrm{W}$ \\
\hline 68 & RV Jurubatuba Mirin & $23^{\circ} 51^{\prime} 27,1^{\prime \prime} \mathrm{S}$ & $46^{\circ} 17^{\prime} 26,0^{\prime \prime} \mathrm{W}$ \\
\hline 69 & RV Mambu & $24^{\circ} 02^{\prime} 00,5^{\prime \prime} \mathrm{S}$ & $46^{\circ} 49^{\prime} 22,6^{\prime \prime} \mathrm{W}$ \\
\hline 70 & RV Aguapeú & $24^{\circ} 06^{\prime} 52,4^{\prime \prime} \mathrm{S}$ & $46^{\circ} 47^{\prime} 19,3^{\prime \prime} \mathrm{W}$ \\
\hline 71 & Rio Cabuçú & $24^{\circ} 19^{\prime} 27,5^{\prime \prime} \mathrm{S}$ & $47^{\circ} 04^{\prime} 49,8^{\prime \prime} \mathrm{W}$ \\
\hline 72 & Re Rio das Pedras & $23^{\circ} 51^{\prime} 24,7 \prime \mathrm{S}$ & $46^{\circ} 27^{\prime} 52,5^{\prime \prime} \mathrm{W}$ \\
\hline \multicolumn{4}{|c|}{ UHGRH 8- Sapucaí / Grande } \\
\hline 73 & He Estreito & $20^{\circ} 09^{\prime} 454^{\prime} \mathrm{S}$ & $47^{\circ} 15^{\prime} 934^{\prime} \mathrm{W}$ \\
\hline 74 & He Jaguará & $20^{\circ} 04^{\prime} 755^{\prime} \mathrm{S}$ & $47^{\circ} 24^{\prime} 610^{\prime} \mathrm{W}$ \\
\hline 75 & He Volta Grande & $20^{\circ} 08^{\prime} 669^{\prime} \mathrm{S}$ & $48^{\circ} 02^{\prime} 343^{\prime} \mathrm{W}$ \\
\hline 76 & He Igaratá & $20^{\circ} 07^{\prime} 669^{\prime} \mathrm{S}$ & $49^{\circ} 02^{\prime} 343^{\prime} \mathrm{W}$ \\
\hline \multicolumn{4}{|c|}{ UHGRH 9 - Mogi-Guaçú } \\
\hline 77 & RV Elektro- Cach. de Emas & $21^{\circ} 58.982^{\prime} \mathrm{S}$ & $47^{\circ} 52.682^{\prime} \mathrm{W}$ \\
\hline 78 & Re dos Biguás & $25^{\circ} 08.555^{\prime} \mathrm{S}$ & $52^{\circ} 01.528^{\prime} \mathrm{W}$ \\
\hline 79 & Re Urbano & $19^{\circ} 59.500^{\prime} \mathrm{S}$ & $49^{\circ} 23.900^{\prime} \mathrm{W}$ \\
\hline 80 & Re do David & $22^{\circ} 19.433^{\prime} \mathrm{S}$ & $46^{\circ} 45.446^{\prime} \mathrm{W}$ \\
\hline 81 & Re Fazenda Aurora & $20^{\circ} 59.820^{\prime} \mathrm{S}$ & $47^{\circ} 58.946^{\prime} \mathrm{W}$ \\
\hline 82 & L. do Diogo & $21^{\circ} 37.450^{\prime} \mathrm{S}$ & $47^{\circ} 48.403^{\prime} \mathrm{W}$ \\
\hline 83 & Re Cristal & $21^{\circ} 36.414^{\prime} \mathrm{S}$ & $47^{\circ} 47.948^{\prime} \mathrm{W}$ \\
\hline 84 & Re Santa Margarida & $21^{\circ} 27.555^{\prime} \mathrm{S}$ & $48^{\circ} 02.020^{\prime} \mathrm{W}$ \\
\hline 85 & L do Barro Preto & $21^{\circ} 29.638^{\prime} \mathrm{S}$ & $48^{\circ} 01.987^{\prime} \mathrm{W}$ \\
\hline 86 & L das Cabras & $21^{\circ} 29.149^{\prime} \mathrm{S}$ & $48^{\circ} 03.723^{\prime} \mathrm{W}$ \\
\hline 87 & L do Peixe & $21^{\circ} 37.420^{\prime} \mathrm{S}$ & $47^{\circ} 48.403^{\prime} \mathrm{W}$ \\
\hline 88 & L Verde & $21^{\circ} 20.624^{\prime} \mathrm{S}$ & $48^{\circ} 07.125^{\prime} \mathrm{W}$ \\
\hline 89 & Re Urbano & $21^{\circ} 37.420^{\prime} \mathrm{S}$ & $47^{\circ} 48.403^{\prime} \mathrm{W}$ \\
\hline
\end{tabular}


Tabela 2. Continuação...

\begin{tabular}{|c|c|c|c|}
\hline & Corpos de água & Latitude & Longitude \\
\hline 90 & Re 2J (Usina Santa Adélia) & $21^{\circ} 55.906^{\prime} \mathrm{S}$ & $48^{\circ} 04.739^{\prime} \mathrm{W}$ \\
\hline 91 & Re do Paço Municipal & $21^{\circ} 05.016^{\prime} \mathrm{S}$ & $48^{\circ} 33.530^{\prime} \mathrm{W}$ \\
\hline 92 & Re São Geraldo & $22^{\circ} 19.434^{\prime} \mathrm{S}$ & $46^{\circ} 45.446^{\prime} \mathrm{W}$ \\
\hline 93 & Re Fazenda Sobrado II & $22^{\circ} 00.784^{\prime} \mathrm{S}$ & $47^{\circ} 10.097^{\prime} \mathrm{W}$ \\
\hline 94 & Re Interlagos & $22^{\circ} 03.362^{\prime} \mathrm{S}$ & $46^{\circ} 59.043^{\prime} \mathrm{W}$ \\
\hline 95 & Re da Praça Basílio re.Seschini & $21^{\circ} 56.060^{\prime} \mathrm{S}$ & $46^{\circ} 42.947^{\prime} \mathrm{W}$ \\
\hline 96 & Re da Churrascaria & $21^{\circ} 57.289^{\prime} \mathrm{S}$ & $46^{\circ} 43.218^{\prime} \mathrm{W}$ \\
\hline 97 & Re Irmãos Ribeiro & $22^{\circ} 10.016^{\prime} \mathrm{S}$ & $46^{\circ} 46.717^{\prime} \mathrm{W}$ \\
\hline 98 & Re Municipal Íria Mota Silva & $22^{\circ} 10.782^{\prime} \mathrm{S}$ & $46^{\circ} 44.627^{\prime} \mathrm{W}$ \\
\hline 99 & Re Churrascaria Sto Antonio & $22^{\circ} 33.866^{\prime} \mathrm{S}$ & $46^{\circ} 32.313^{\prime} \mathrm{W}$ \\
\hline 100 & Re Ivo Carotini & $22^{\circ} 27.959^{\prime} \mathrm{S}$ & $46^{\circ} 37.660^{\prime} \mathrm{W}$ \\
\hline 101 & Re dos Macaquinhos & $22^{\circ} 36.901^{\prime} \mathrm{S}$ & $46^{\circ} 44.272^{\prime} \mathrm{W}$ \\
\hline 102 & Re Jovino Silveira & $22^{\circ} 37.892^{\prime} \mathrm{S}$ & $46^{\circ} 43.630^{\prime} \mathrm{W}$ \\
\hline 103 & Re. CEPTA & $22^{\circ} 01.982^{\prime} \mathrm{S}$ & $47^{\circ} 52.682^{\prime} \mathrm{W}$ \\
\hline 104 & Re Mogi-Guaçu & $22^{\circ} 22.935^{\prime} \mathrm{S}$ & $46^{\circ} 53.935^{\prime} \mathrm{W}$ \\
\hline 105 & Re Lago Municipal & $22^{\circ} 21.638^{\prime} \mathrm{S}$ & $47^{\circ} 23.009^{\prime} \mathrm{W}$ \\
\hline \multicolumn{4}{|c|}{ UHGRH 10- Sorocaba } \\
\hline 106 & Re Itupararanga & $23^{\circ} 35.813^{\prime} \mathrm{S}$ & $047^{\circ} 18.075^{\prime} \mathrm{W}$ \\
\hline 107 & Re Prainha & $23^{\circ} 34.894^{\prime} \mathrm{S}$ & $047^{\circ} 26.045^{\prime} \mathrm{W}$ \\
\hline 108 & Re Hedberg & $23^{\circ} 25.607^{\prime} \mathrm{S}$ & $047^{\circ} 35.678^{\prime} \mathrm{W}$ \\
\hline \multicolumn{4}{|c|}{ UHGRH 11- Ribeira do Iguape } \\
\hline 109 & Rio Rib. Iguape & $24^{\circ} 35^{\prime} 25,2^{\prime \prime} \mathrm{S}$ & $48^{\circ} 35^{\prime} 44,7^{\prime \prime} \mathrm{W}$ \\
\hline 110 & Re Cong.Cris. no Brasil & $24^{\circ} 33^{\prime} 01,6$ " S & $48^{\circ} 08^{\prime} 04,1^{\prime \prime} \mathrm{W}$ \\
\hline 111 & Re de Juquiazinho & $23^{\circ} 56^{\prime} 00,5^{\prime \prime} \mathrm{S}$ & $47^{\circ} 30^{\prime} 25,0^{\prime \prime} \mathrm{W}$ \\
\hline 112 & He do Porto Raso & $24^{\circ} 03^{\prime} 30,6^{\prime \prime} \mathrm{S}$ & $47^{\circ} 24^{\prime} 35,1^{\prime \prime} \mathrm{W}$ \\
\hline 113 & He da Serraria & $24^{\circ} 08^{\prime} 43,2 ” \mathrm{~S}$ & $47^{\circ} 32^{\prime} 27,8^{\prime \prime} \mathrm{W}$ \\
\hline 114 & He Alecrim & $24^{\circ} 04^{\prime} 46,1^{\prime \prime} \mathrm{S}$ & $47^{\circ} 28^{\prime} 34,1^{\prime \prime} \mathrm{W}$ \\
\hline 115 & He Cach. da França & $23^{\circ} 56^{\prime} 04,4^{\prime \prime} S$ & $47^{\circ} 11^{\prime} 20,7^{\prime \prime} \mathrm{W}$ \\
\hline 116 & He fumaça & $24^{\circ} 00^{\prime} 16,2 " \mathrm{~S}$ & $47^{\circ} 15^{\prime} 40,6^{\prime \prime} \mathrm{W}$ \\
\hline 117 & He Barra & $24^{\circ} 000^{\prime} 00,5^{\prime \prime} \mathrm{S}$ & $47^{\circ} 20^{\prime} 37,3^{\prime \prime} \mathrm{W}$ \\
\hline 118 & $\mathrm{He}$ do Iporanga-CBA & $24^{\circ} 06^{\prime} 08,2^{\prime \prime} \mathrm{S}$ & $47^{\circ} 43^{\prime} 48,1^{\prime \prime} \mathrm{W}$ \\
\hline 119 & He. de Jurupará & $23^{\circ} 57^{\prime} 19,0$ 's & $47^{\circ} 23^{\prime} 58,0^{\prime \prime} \mathrm{W}$ \\
\hline \multicolumn{4}{|c|}{ UHGRH 12- baixo pardo/Grande } \\
\hline 120 & Re Urbano & $20^{\circ} 46^{\prime} 32,3^{\prime \prime} \mathrm{SS}$ & $48^{\circ} 27^{\prime} 03,0 ” \mathrm{~W}$ \\
\hline 121 & Rio do Turvo & $20^{\circ} 40^{\prime} 40,6^{\prime \prime} \mathrm{SS}$ & $48^{\circ} 30^{\prime} 40,1^{\prime \prime} \mathrm{W}$ \\
\hline 122 & Re Cestari & $20^{\circ} 33^{\prime} 53,4^{\prime \prime} \mathrm{S}$ & $48^{\circ} 34^{\prime} 46,2^{\prime \prime} \mathrm{W}$ \\
\hline 123 & He Porto Colômbia & $20^{\circ} 07^{\prime} 35,5^{\prime \prime} \mathrm{S}$ & $48^{\circ} 33^{\prime} 43,3^{\prime \prime} \mathrm{W}$ \\
\hline 124 & Rio Foz do Rio Pardo & $20^{\circ} 10^{\prime} 23,2 ” S$ & $48^{\circ} 37^{\prime} 41,4^{\prime \prime} \mathrm{W}$ \\
\hline 125 & Re dos Soppa & $20^{\circ} 11^{\prime} 12,3 ” S$ & $48^{\circ} 38^{\prime} 59,9^{\prime \prime} \mathrm{W}$ \\
\hline 126 & He Marimbondo & $20^{\circ} 18^{\prime} 30,1^{\prime \prime} \mathrm{S}$ & $49^{\circ} 10^{\prime} 29,4^{\prime \prime} \mathrm{W}$ \\
\hline \multicolumn{4}{|c|}{ UHGRH 13- Jacaré / Tiete } \\
\hline 127 & Re Jardim Botânico & $22^{\circ} 20.304^{\prime} \mathrm{S}$ & $49^{\circ} 00.866^{\prime} \mathrm{W}$ \\
\hline 128 & Re Zoológico & $22^{\circ} 20.515^{\prime} \mathrm{S}$ & $49^{\circ} 01.060^{\prime} \mathrm{W}$ \\
\hline 129 & Re Rio Batalha & $22^{\circ} 22.875^{\prime} \mathrm{S}$ & $49^{\circ} 06.924^{\prime} \mathrm{W}$ \\
\hline 130 & Re Secretaria da Agricultura & $22^{\circ} 21.340^{\prime} \mathrm{S}$ & $48^{\circ} 45.387^{\prime} \mathrm{W}$ \\
\hline 131 & Re da Fazenda Primavera & $22^{\circ} 07.724^{\prime} \mathrm{S}$ & $47^{\circ} 51.630^{\prime} \mathrm{W}$ \\
\hline 132 & Re Instituto Florestal & $22^{\circ} 14.864^{\prime} \mathrm{S}$ & $47^{\circ} 49.421^{\prime} \mathrm{W}$ \\
\hline 133 & Re da Areia que Canta & $22^{\circ} 18.904^{\prime} \mathrm{S}$ & $48^{\circ} 03.072^{\prime} \mathrm{W}$ \\
\hline 134 & Re Fazenda Sonho Meu I & $22^{\circ} 24.902^{\prime} \mathrm{S}$ & $48^{\circ} 14.353^{\prime} \mathrm{W}$ \\
\hline 135 & Re Sítio Mariano Lopes & $22^{\circ} 23.317^{\prime} \mathrm{S}$ & $48^{\circ} 22.859^{\prime} \mathrm{W}$ \\
\hline 136 & Re Marisa (Usina Tamoio) & $21^{\circ} 55.523^{\prime} \mathrm{S}$ & $48^{\circ} 06.590^{\prime} \mathrm{W}$ \\
\hline
\end{tabular}


Tabela 2. Continuação...

\begin{tabular}{|c|c|c|c|}
\hline & Corpos de água & Latitude & Longitude \\
\hline 137 & Re Faz. Méia Ribeirão Itaquerê & $21^{\circ} 47.654^{\prime} \mathrm{S}$ & $48^{\circ} 33.896^{\prime} \mathrm{W}$ \\
\hline 138 & Re Fazenda Palmeiras & $21^{\circ} 45.708^{\prime} \mathrm{S}$ & $48^{\circ} 41.953^{\prime} \mathrm{W}$ \\
\hline 139 & Re Sítio Boa Vista & $21^{\circ} 47.281^{\prime} \mathrm{S}$ & $48^{\circ} 47.831^{\prime} \mathrm{W}$ \\
\hline 140 & He Broa & $22^{\circ} 10.224^{\prime} \mathrm{S}$ & $47^{\circ} 54.244^{\prime} \mathrm{W}$ \\
\hline 141 & $\operatorname{Re} 29$ & $21^{\circ} 53.742^{\prime} \mathrm{S}$ & $47^{\circ} 49.034^{\prime} \mathrm{W}$ \\
\hline 142 & Re do Chile & $21^{\circ} 52.024^{\prime} \mathrm{S}$ & $47^{\circ} 51.917^{\prime} \mathrm{W}$ \\
\hline 143 & Re do Clube Náutico & $21^{\circ} 42.405^{\prime} \mathrm{S}$ & $48^{\circ} 01.614^{\prime} \mathrm{W}$ \\
\hline 144 & He Jacaré Pepira & $22^{\circ} 05.224^{\prime} \mathrm{S}$ & $46^{\circ} 54.244^{\prime} \mathrm{W}$ \\
\hline 145 & He Bariri & $22^{\circ} 09.728^{\prime} \mathrm{S}$ & $48^{\circ} 44.560^{\prime} \mathrm{W}$ \\
\hline 146 & He Barra Bonita & $22^{\circ} 31.818^{\prime} \mathrm{S}$ & $48^{\circ} 31.231^{\prime} \mathrm{W}$ \\
\hline
\end{tabular}

UHGRHs 14, 17 \& 22 Alto Paranapanema, Médio Paranapanema e pontal do Paranapanema

\begin{tabular}{|c|c|c|c|}
\hline 147 & He Jurumirim & $23^{\circ} 13.38^{\prime} \mathrm{S}$ & $49^{\circ} 13 \cdot 30,7^{\prime} \mathrm{W}$ \\
\hline 148 & He Chavantes & $23^{\circ} 826,7^{\prime} \mathrm{S}$ & $49^{\circ} 42.24,3^{\prime} \mathrm{W}$ \\
\hline 149 & He Canoas I & $22^{\circ} 56.30,7^{\prime} \mathrm{S}$ & $50^{\circ} 30.41,3^{\prime} \mathrm{W}$ \\
\hline 150 & He Salto Grande & $22^{\circ} 53.53^{\prime} \mathrm{S}$ & $49^{\circ} 59.32,5^{\prime} \mathrm{W}$ \\
\hline 151 & He Capivara & $22^{\circ} 39.3,3^{\prime} \mathrm{S}$ & $51^{\circ} 20.27^{\prime} \mathrm{W}$ \\
\hline 152 & He Taquaruçu & $22^{\circ} 32.36,4^{\prime} \mathrm{S}$ & $51^{\circ} 59.18,6^{\prime} \mathrm{W}$ \\
\hline 153 & He Rosana & $22^{\circ} 35.40^{\prime} \mathrm{S}$ & $52^{\circ} 51.13,8^{\prime} \mathrm{W}$ \\
\hline \multicolumn{4}{|c|}{ UHGRH 15- Turvo/ Grande } \\
\hline 154 & Re Rio Grande & $21^{\circ} 16^{\prime} 11,6^{\prime \prime} \mathrm{S}$ & $48^{\circ} 31^{\prime} 42,2^{\prime \prime} \mathrm{W}$ \\
\hline 155 & Re Faz.Sucesso & $20^{\circ} 57^{\prime} 48,5^{\prime \prime} \mathrm{S}$ & $48^{\circ} 40^{\prime} 45,3^{\prime \prime} \mathrm{W}$ \\
\hline 156 & Re Sítio Liberdade & $20^{\circ} 52 ’ 34,1^{\prime \prime} \mathrm{S}$ & $48^{\circ} 40^{\prime} 31,2^{\prime \prime} \mathrm{W}$ \\
\hline 157 & Re Tabapuã & $20^{\circ} 56^{\prime} 02,3^{\prime \prime} \mathrm{S}$ & $49^{\circ} 05^{\prime} 15,8^{\prime \prime} \mathrm{W}$ \\
\hline 158 & Re.São Domingos & $21^{\circ} 05^{\prime} 37,8^{\prime \prime} \mathrm{S}$ & $49^{\circ} 01^{\prime} 20,4$ " W \\
\hline 159 & Re Santana & $20^{\circ} 56^{\prime} 24,9^{\prime \prime} \mathrm{S}$ & $49^{\circ} 15^{\prime} 29,0^{\prime \prime} \mathrm{W}$ \\
\hline 160 & Re Fazenda Brasil & $20^{\circ} 45^{\prime} 45,8^{\prime \prime} \mathrm{S}$ & $49^{\circ} 32^{\prime} 58,4^{\prime \prime} \mathrm{W}$ \\
\hline 161 & Re Chác. Pomarco & $20^{\circ} 48^{\prime} 51,4^{\prime \prime} \mathrm{S}$ & $49^{\circ} 28^{\prime} 55,1^{\prime \prime} \mathrm{W}$ \\
\hline 162 & Re Rio Preto & $20^{\circ} 51^{\prime} 37,1^{\prime \prime} \mathrm{S}$ & $49^{\circ} 20^{\prime} 08,2^{\prime \prime} \mathrm{W}$ \\
\hline 163 & Re Tanabi & $20^{\circ} 32^{\prime} 40,6^{\prime \prime} \mathrm{S}$ & $49^{\circ} 37^{\prime} 40,0^{\prime \prime} \mathrm{W}$ \\
\hline 164 & Re Ibiporanga & $20^{\circ} 24^{\prime} 59,8^{\prime \prime} \mathrm{S}$ & $49^{\circ} 29^{\prime} 10,3^{\prime \prime} \mathrm{W}$ \\
\hline 165 & He Água Vermelha & $19^{\circ} 53^{\prime} 06,6^{\prime \prime} \mathrm{S}$ & $50^{\circ} 18^{\prime} 32,9 ” \mathrm{~W}$ \\
\hline 166 & Re Urânia & $20^{\circ} 14^{\prime} 27,7^{\prime \prime} \mathrm{S}$ & $50^{\circ} 36^{\prime} 57,6^{\prime \prime} \mathrm{W}$ \\
\hline \multicolumn{4}{|c|}{ UHGRH 16- Tietê Batalha } \\
\hline 167 & Re Rio das Pedras & $21^{\circ} 50^{\prime} 49,8^{\prime \prime}$ & $21^{\circ} 57^{\prime} 23,4^{\prime \prime}$ \\
\hline 168 & Rio Tietê & $21^{\circ} 50^{\prime} 36,4^{\prime \prime}$ & $48^{\circ} 57^{\prime} 14,7^{\prime \prime}$ \\
\hline 169 & Re Borborema & $21^{\circ} 39^{\prime} 31,4^{\prime \prime}$ & $49^{\circ} 08^{\prime} 36,3^{\prime \prime}$ \\
\hline 170 & Re Rio Formoso & $21^{\circ} 21^{\prime} 44,4^{\prime \prime}$ & $49^{\circ} 23^{\prime} 07,5^{\prime \prime}$ \\
\hline 171 & He Promissão & $21^{\circ} 20^{\prime} 33,6^{\prime \prime}$ & $49^{\circ} 44^{\prime} 37,2^{\prime \prime}$ \\
\hline 172 & He Ibitinga & $21^{\circ} 50^{\prime} 49,8^{\prime \prime} \mathrm{S}$ & $48^{\circ} 57^{\prime} 23,4^{\prime \prime} \mathrm{W}$ \\
\hline 173 & Re Faz. Sta. Catarina & $21^{\circ} 44^{\prime} 07,9^{\prime \prime} \mathrm{S}$ & $48^{\circ} 55^{\prime} 47,6^{\prime \prime} \mathrm{W}$ \\
\hline 174 & He Promissão & $21^{\circ} 20^{\prime} 33,6^{\prime \prime} \mathrm{S}$ & $49^{\circ} 44^{\prime} 37,2^{\prime \prime} \mathrm{W}$ \\
\hline \multicolumn{4}{|c|}{ UHGRH 18- S. Jose dos Dourados } \\
\hline 175 & He Ilha Solteira & $20^{\circ} 22^{\prime} 57,9^{\prime \prime} \mathrm{S}$ & $51^{\circ} 20^{\prime} 11,9^{\prime \prime} \mathrm{W}$ \\
\hline 176 & Re Estância Semax & $20^{\circ} 33^{\prime} 11,6^{\prime \prime} \mathrm{S}$ & $50^{\circ} 01 ’ 37,6 ” \mathrm{~W}$ \\
\hline 177 & LM SP-310 & $20^{\circ} 39^{\prime} 04,4^{\prime \prime} \mathrm{S}$ & $50^{\circ} 27^{\prime} 11,0^{\prime \prime} \mathrm{W}$ \\
\hline 178 & Re Sítio São Pedro & $20^{\circ} 42^{\prime} 39,5^{\prime \prime} \mathrm{S}$ & $49^{\circ} 52^{\prime} 05,6^{\prime \prime} \mathrm{W}$ \\
\hline 179 & LM Monte Aprazível & $20^{\circ} 45^{\prime} 18,0^{\prime \prime} \mathrm{S}$ & $49^{\circ} 43^{\prime} 20,2^{\prime \prime} \mathrm{W}$ \\
\hline \multicolumn{4}{|c|}{ UHGRH 19- Baixo Tietê } \\
\hline 180 & He Nova Avanhandava & $21^{\circ} 06^{\prime} 21,3^{\prime \prime}$ & $50^{\circ} 10^{\prime} 55,8^{\prime \prime}$ \\
\hline 181 & LM Birigui & $21^{\circ} 14^{\prime} 47,2^{\prime \prime}$ & $50^{\circ} 23^{\prime} 40,8^{\prime \prime}$ \\
\hline
\end{tabular}


Tabela 2. Continuação...

\begin{tabular}{|c|c|c|c|}
\hline & Corpos de água & Latitude & Longitude \\
\hline 182 & L SP-463 & $21^{\circ} 04^{\prime} 41,5^{\prime \prime}$ & $50^{\circ} 27^{\prime} 49,7^{\prime \prime}$ \\
\hline 183 & L Araçatuba & $21^{\circ} 12^{\prime} 03,4^{\prime \prime}$ & $50^{\circ} 32^{\prime} 42,3 \prime$ \\
\hline 184 & L Rondon - Km 580 & $21^{\circ} 11^{\prime} 44,3^{\prime \prime}$ & $50^{\circ} 53^{\prime} 52,0^{\prime \prime}$ \\
\hline 185 & He Jupiá & $20^{\circ} 45^{\prime} 09,3^{\prime \prime}$ & $51^{\circ} 37^{\prime} 20,7^{\prime \prime}$ \\
\hline 186 & He Três Irmãos & $20^{\circ} 40^{\prime} 16,0^{\prime \prime}$ & $51^{\circ} 17^{\prime} 00,0^{\prime \prime}$ \\
\hline 187 & LM Rondon - Km 628 & $20^{\circ} 57^{\prime} 03,9^{\prime \prime}$ & $51^{\circ} 16^{\prime} 41,9^{\prime \prime}$ \\
\hline \multicolumn{4}{|c|}{ UHGRH 20 - Aguapeí } \\
\hline 188 & Re urbano & $22^{\circ} 12^{\prime} 56,6^{\prime \prime} \mathrm{S}$ & $49^{\circ} 38^{\prime} 44,6^{\prime \prime} \mathrm{W}$ \\
\hline 189 & Re Santa Thereza & $22^{\circ} 12^{\prime} 11,8^{\prime \prime} \mathrm{S}$ & $49^{\circ} 43^{\prime} 49,7^{\prime \prime} \mathrm{W}$ \\
\hline 190 & Re 7 de Setembro & $21^{\circ} 51^{\prime} 44,5^{\prime \prime} \mathrm{S}$ & $50^{\circ} 32^{\prime} 09,8^{\prime \prime} \mathrm{W}$ \\
\hline 191 & Re Country Clube & $21^{\circ} 56^{\prime} 48,2^{\prime \prime} \mathrm{S}$ & $50^{\circ} 25^{\prime} 12,2^{\prime \prime} \mathrm{W}$ \\
\hline 192 & Re Rio Aguapeí & $21^{\circ} 42^{\prime} 24,4^{\prime \prime} \mathrm{S}$ & $50^{\circ} 30^{\prime} 47,8^{\prime \prime} \mathrm{W}$ \\
\hline 193 & LM A. Chateaubriand & $21^{\circ} 41^{\prime} 00,9^{\prime \prime} \mathrm{S}$ & $50^{\circ} 36^{\prime} 34,9^{\prime \prime} \mathrm{W}$ \\
\hline 194 & Rio Paraná & $21^{\circ} 20^{\prime} 52,9^{\prime \prime} \mathrm{S}$ & $51^{\circ} 51^{\prime} 45,0^{\prime \prime} \mathrm{W}$ \\
\hline 195 & L central - R. Paraná & $21^{\circ} 17^{\prime} 44,6^{\prime \prime} \mathrm{S}$ & $51^{\circ} 51^{\prime} 02,1^{\prime \prime} \mathrm{W}$ \\
\hline 196 & L central -R. Paraná 2 & $21^{\circ} 14^{\prime} 35,9^{\prime \prime} \mathrm{S}$ & $51^{\circ} 51^{\prime} 07,3$ ” W \\
\hline 197 & LM do Pau da Onça & $21^{\circ} 05^{\prime} 15,8^{\prime \prime} \mathrm{S}$ & $51^{\circ} 42^{\prime} 42,1^{\prime \prime} \mathrm{W}$ \\
\hline 198 & LM do Marreco & $21^{\circ} 06^{\prime} 04,1^{\prime \prime} \mathrm{S}$ & $51^{\circ} 43^{\prime} 53,3^{\prime \prime} \mathrm{W}$ \\
\hline 199 & Rio Foz do Aguapei & $21^{\circ} 03,04,0 " \mathrm{~S}$ & $51^{\circ} 45^{\prime} 58,4^{\prime \prime} \mathrm{W}$ \\
\hline \multicolumn{4}{|c|}{ UHGRH 21- Peixe } \\
\hline 200 & Re Municipal Cascata & $22^{\circ} 12^{\prime} 52,2^{\prime \prime} \mathrm{S}$ & $49^{\circ} 55^{\prime} 31,9^{\prime \prime} \mathrm{W}$ \\
\hline 201 & Re Fazenda 3 Rios & $22^{\circ} 17^{\prime} 48,8^{\prime \prime} \mathrm{S}$ & $49^{\circ} 59^{\prime} 33,5^{\prime \prime} \mathrm{W}$ \\
\hline 202 & Re Fazenda Jabuti & $21^{\circ} 47^{\prime} 26,7^{\prime \prime} \mathrm{S}$ & $51^{\circ} 03^{\prime} 51,0 ” \mathrm{~W}$ \\
\hline 203 & LM dos Patos & $21^{\circ} 44^{\prime} 25,3^{\prime \prime} \mathrm{S}$ & $51^{\circ} 22^{\prime} 33,5^{\prime \prime} \mathrm{W}$ \\
\hline 204 & LM Rio do Peixe & $21^{\circ} 46^{\prime} 12,9^{\prime \prime} \mathrm{S}$ & $51^{\circ} 21^{\prime} 04,1^{\prime \prime} \mathrm{W}$ \\
\hline 205 & Re Nasc. do Rib. Claro & $21^{\circ} 57^{\prime} 03,0^{\prime \prime} \mathrm{S}$ & $51^{\circ} 37^{\prime} 48,2^{\prime \prime} \mathrm{W}$ \\
\hline 206 & Rio do Peixe & $21^{\circ} 32^{\prime} 41,0^{\prime \prime} \mathrm{S}$ & $51^{\circ} 57^{\prime} 44,7^{\prime \prime} \mathrm{W}$ \\
\hline
\end{tabular}

na sua maioria de lagoas temporárias, áreas inundadas e campos úmidos e estas não foram encontradas nos corpos de água do Estado de São Paulo.

No Estado de São Paulo, tanto as represas de grande porte ou de pequeno, tiveram uma grande importância na riqueza de espécies dos Cyclopoida. Entretanto, estes reservatórios construídos principalmente para geração de energia elétrica são interligados, promovendo uma homogeneização das comunidades planctônicas. Silva (2008) observou que nos reservatórios em cascata as espécies de Cyclopoida tendem ser as mesmas, variando apenas a sua densidade e dominância, segundo as características morfométricas e de qualidade de água dos reservatórios. Por outro lado, o Estado de São Paulo é destituído de lagos naturais permanentes e a construção de reservatórios promove o aumento de novos habitats para as populações de Copepoda Cyclopoida e redesenhando a distribuição destes organismos no estado.

\section{Comentários sobre riqueza de espécies no estado de são paulo comparado com outras regiões}

Assim como acontece com outros grupos zooplanctônicos o grupo dos Copepoda Cyclopoida foi bem estudado em corpos de água da região amazônica (Estado do Amazonas e Pará), com a ocorrência de espécies que não foram encontradas nos corpos de água do Estado de São Paulo. Como no Estado de São Paulo foram identificadas 39 espécies do total de 84 espécies encontradas no Brasil, poderia pensar que em termos de área explorada, o Estado de São Paulo apresenta maior riqueza de espécies, em relação as outras áreas, porém, isto não é verdade pois devemos levar em consideração que o numero de especialistas dedicando-se ao estudo dos grupos taxonômicos zooplanctônicos é maior no Estado de São Paulo do que nas outras regiões.

\section{Principais avanços relacionados ao Programa BIOTA/FAPESP}

No projeto "Biodiversidade zooplanctônica e o estado de degradação dos ecossistemas aquáticos continentais do Estado de São Paulo" foram amostrados 207 corpos de água nas 22 unidades hídricas de gerenciamento de recursos hídricos (UGRHI) e grande parte das espécies de Copepoda Cyclopoida de vida livre de água doce anteriormente registradas foram encontradas, sendo que todas as planctônicas ocorreram.

O Estado de São Paulo tem registrado $46 \%$ das espécies de Copepoda Cyclopoida de vida livre de água doce conhecidas no Brasil. Foram registradas 39 espécies no Estado, englobando tanto espécies de hábito planctônico quanto as não planctônicas. Sendo que, seis novos registros foram oriundos do Programa BIOTA/FAPESP, incluindo uma espécie nova, o Thermocyclops iguapensis, uma espécie restrita a costa atlântica (Silva \& Matsumura-Tundisi 2005a).

O Programa BIOTA/FAPESP proporcionou também a correção de erros de identificação, como para as espécies Mesocyclops ogunnus identificada como M. kieferi, para M. meridianus identificada como M. brasilianus e Paracyclops chiltoni identificada como P. fimbriatus. Estas correções proporcionam um melhor conhecimento 
da ecologia da espécie assim como do sistema, como verificado por Silva \& Matsumura-Tundisi (2002) para relações entre espécies de Cyclopoida e o grau de trofia de reservatórios do médio e baixo Tietê.

Além do avanço na taxonomia tradicional (morfológica) houve também a implantação de técnicas de citotaxonomia, que permitiu a separação de espécies sul americanas de Mesocyclops longisetus das norte americanas (Silva \& Matsumura-Tundisi 2004) e de taxonomia molecular (Silva \& Matsumura-Tundisi 2005b), ambas inéditas no Brasil para aplicação neste grupo.

\section{Principais grupos de pesquisa}

No Estado de São Paulo atualmente não existe grupo de pesquisa em Biodiversidade de zooplâncton que possa dar continuidade ao trabalho desenvolvido no BIOTA/FAPESP, pois após o término do Programa BIOTA/FAPESP, os pesquisadores se dispersaram, e aqueles vinculados à docência das Universidades não conseguiram dar prosseguimento à pesquisa em grupos taxonômicos. E os estudantes formados (doutores e pós doutores) também não tiveram oportunidade de se inserirem em Instituições de pesquisa para dar continuidade à pesquisa ficando totalmente perdidos.

\section{Principais acervos}

Os acervos principais se concentram em São Carlos, SP, em duas instituições, o Instituto Internacional de Ecologia que conta com amostras do Programa BIOTA/FAPESP (1999-2003), que amostrou todas as unidades hidrográficas de São Paulo e também dispõe de amostras de DNA extraídas de algumas espécies. A outra instituição é o Departamento de Ecologia Evolutiva da Universidade Federal de São Carlos, SP, que conta com amostras tanto do BIOTA/FAPESP (1999-2003) quanto do projeto Tipologia de represas (1978-1981), que abrangeram grande parte do Estado de São Paulo, além de amostras de várias regiões do Brasil. O Museu de Zoologia da Universidade de São Paulo também tem um acervo que contém muitos tipos da fauna de Copepoda Cyclopoida descrita no Estado de São Paulo.

\section{Principais lacunas do conhecimento}

As principais lacunas são a não continuidade na formação de especialistas que abordem novas técnicas taxonômicas e de falta de conhecimento da biologia e ciclo de vida de grande parte das espécies. E também a formação de especialistas em várias regiões do Brasil que se dediquem à biodiversidade dos grupos taxonômicos, e só assim podemos ter uma visão mais precisa da riqueza de espécies que ocorrem no Brasil.

\section{Perspectivas de pesquisa nos próximos 10 anos}

Deve haver uma maior integração entre as unidades brasileiras para ampliar o conhecimento da distribuição e consequentemente da ecologia das espécies e do sistema. A implantação de um banco genético para comparar espécies seria de grande importância para a taxonomia de Copepoda Cyclopoida. E elaboração de modelos de dispersão em reservatórios considerando as características físicas e químicas do ambiente.

\section{Referências bibliográficas}

ARCIFA, M.S. 1984. Zooplankton composition of ten reservoirs in southern Brazil. Hydrobiologia 113:137-145. http://dx.doi.org/10.1007/ BF00026600

CLAUS, C. 1893. Neue Beobachtungen über die Organisation und Entwicklung von Cyclops Ein Beitrag zur Systematik der Cyclopiden. Arb. Inst. Wien 10:238-356.

DUSSART, B.H. \& DEFAYE, D. 1985. Reportoire Mondial des Copépodes Cyclopoides. Editions du C.N.R.S., Paris, 236p.
DUSSART, B.H. 1984. Some Crustacea Copepoda from Venezuela. Hydrobiologia 113:25-67. http://dx.doi.org/10.1007/BF00026592

DUSSART, B.H. 1987. Sur quelques Mesocyclops (Crustacea, Copepoda) d'Amérique du Sud. Amazoniana 10(2):149-161.

GUTIERREZ-AGUIRRE, M. \& SUAREZ-MORALES, E. 2001. Distributions and taxonomy of the tropical American Mesocyclops G.O. Sar, 1914. Crustaceana 74:477-487. http://dx.doi.org/10.1163/156854001750243054

HUYS, R. \& BOXSHALL, G. A. 1991. Copepod evolution. The Ray Society, London, 468p.

KARAYTUG, S. 1999. Copepoda: Cyclopoida. Genera Paracyclops and key to the Eucyclopinae. In Guides to the identification of the Microinvertebrates of the continental Waters of the World (J.H. Dumont, ed.). Backhuys Publishers.

KIEFER, F. 1925. Eine neuer Süsswasser-copepod aus Sud-Amerika. Zool. Anz. 63.

KIEFER, F. 1927. Versuch eines System der Cyclopiden. Zool. Anz. 72(9-10): 400-420.

KIEFER, F. 1929. Crustacea Copepoda. Gnathosoma. Tierreich. 53:1-102.

KIEFER, F. 1931. Die Untergattung Tropocyclops der Gattung Eucyclops (Copepoda, Cyclopoida). Z. Wiss. Zool. 138(3):487-514.

KIEFER, F. 1933. Susswassercopepoden aus Brasilien. Zoologischer Anzeiger 105:38-43.

KIEFER, F. 1936. Brasilianische Ruderfusskrebse (Crustacea Copepoda) gesammelt von Herm Dr. Otto Schubart. Zool. Anz. 116:(1-2):31-35.

KIEFER, F. 1956. Freilebende Ruderfusskrebse (Crustacea Copepoda). I . Calanoida und Cyclopoida. Ergebn. Deutsch. Limnol. Venezuela-Exped. 1:232-268.

KIEFER, F. 1976. Revision der Robustus vernalis- Grupe der gattung Acanthocyclops Kiefer (Crustacea, Copepoda) (Mit eingehender Beurteilung des "Cyclops americanus" Marsh 1975). Bietr. Naturk. Forsch. Sudwestdeutsch. 35:95-110.

KIEFER, F. 1981. Beitrag zur Kenntnis von morphologie, Taxonomie und geographischer verbreitung von Mesocyclops leukarti autorum. Arch. Hydrobiolo. 62(1):148-190. Suppl.

LOWNDES, A.G. 1934. Reports of an expeditions to Brazil and Paraguay in 1926-7 supported by the Trustees of the Percy Sladen Memorial Fund and the executive Committee of the Carniegie Trust of Scotland. Journal of the Linnean Society of London Zoology 39:83-131. http://dx.doi. org/10.1111/j.1096-3642.1934.tb00260.x

MATSUMURA-TUNDISI, T \& ROCHA, O. 1983. Occurrence of Copepods (Calanoida, Cyclopoida and Harpacticoida) from Broa Reservoir (Sao Carlos, Sao Paulo, Brazil). Rev. Bras. Biol. 13(1):1-17.

MATSUMURA-TUNDISI, T. \& SILVA, W.M. 1999. Crustaceos copépodos planctônicos. In Biodiversidade do Estado de São Paulo, Brasil: síntese do conhecimento ao final do século XX. Invertebrados de Água Doce (C.A. Joly \& C.E.M. Bicudo, org.). FAPESP, São Paulo, v.4.

MATSUMURA-TUNDISI, T. \& SILVA, W.M. 2002. Occurrence of Mesocyclops ogunnus Onabamiro, 1957 (Copepoda Cyclopoida) in water bodies of Sao Paulo State, identified as Mesocyclops kieferi Van de Velde, 1984. Braz. J. Biol. 62(4A):615-620. http://dx.doi.org/10.1590/ S1519-69842002000400009

MATSUMURA-TUNDISI, T., RIETZLER, A.C., ESPÍNDOLA, E.L.G. 1990. Predation on Ceriodaphnia cornuta and Brachionus calyciflorus by two Mesocyclops species coexisting in Barra Bonita Reservoir. Hydrobiologia 198:141-151. http://dx.doi.org/10.1007/BF00048631

NOGUEIRA, M.G. 2002. Zooplankton composition, dominance and abundance as a indicator of environmental compartimentalization in Jurumirim Reservoir (Paranapanema River) São Paulo, Brazil. Hydrobiologia 455(1):1-18. http://dx.doi.org/10.1023/A:1011946708757

NOGUEIRA, M.G., JORCIN, A., VIANNA, N.C. \& BRITTO, Y.C. 2004. Reservatórios em cascata e os efeitos na limnologia e organização das comunidades bióticas (fitoplâncton, zooplâncton e zoobentos): Um estudo de caso no rio Paranapanema (SP/PR). In Ecologia de reservatórios: impactos potenciais, ações de manejo e sistemas em cascata (M.G. Nogueira, R. Henry \& A. Jorcin, eds.). Rima, São Carlos, p.435-459. 
REID, J. 1985. Chave de identificação e lista de referências bibliográficas para as espécies continentais sulamericanas de vida livre da ordem Cyclopoida (Crustácea Copepoda). Bol. Zool. 9:17-143.

REID, J. 1989. The distribution of species of the genus Thermocyclops (Copepoda Cyclopoida) in the Western hemisphere, with description of T. parvus, new species. Hydrobiologia 175:149-174. http://dx.doi. org/10.1007/BF00765125

ROCHA, C.E.F \& BOTELHO, M.J.C. 1998. Maxillopda-Copepoda. Cyclopoida. In Catalogue of Crustacea of Brazil (P.S. Young, ed.). Museu Nacional, Rio de Janeiro, p.129-166. (Série Livros, n.6).

ROCHA, C.E.F. 1999. Classe Copepoda (não planctônicos). In Biodiversidade do Estado de São Paulo, Brasil: síntese do conhecimento ao final do século XX. Invertebrados de Água Doce (C.A. Joly \& C.E.M. Bicudo, org.). FAPESP, São Paulo, v.4.

ROCHA, C.E.F.D. \& BJORNBERG, M.H.G.D.C. 1987. Copepods of the Jureia Ecological Reserve, State of Sao Paulo, Brazil: II. The genera Hesperocyclops, Muscocyclops, and Bryocyclops (Cyclopoida, Cyclopidae). Hydrobiologia 153:97-108. http://dx.doi.org/10.1007/ BF00006642

ROCHA, O., SENDACZ, S. \& MATSUMURA-TUNDISI, T. 1995. Composition, biomass and productivity of zooplankton in natural lakes and reservoirs of Brazil. Limnology in Brazil. ABC/SBL, Rio de Janeiro, p.151-166.

SARS, iniciais. 1901. Contributions to the knowledge of the freshwater Entomostraca of South America, 2: Copepoda, Ostracoda. Archiv. Math. Naturvidensk 24(1):1-52.

SENDACZ, S. \& KUBO, E. 1982. Copepoda (Calanoida e Cyclopoida) de reservatórios do Estado de São Paulo. Bol. Inst. Pesca 9:51-189.
SENDACZ, S. 1993. Distribuição geográfica de alguns organismos zooplanctônicos na América do Sul. Acta Limnol. Bras. 6:31-41.

SILVA, W.M. \& MATSUMURA-TUNDISI, T. 2002. Distribution and abundance of Cyclopoida populations in a cascade of reservoir of the Tietê River (São Paulo State, Brazil). Ver. Int. ver. Limnol. 28:667-670.

SILVA, W.M. \& MATSUMURA-TUNDISI, T. 2004. Cytogenetics of the freshwater cyclopoid Mesocyclops longisetus longisetus (Crustacea, Copepoda) from São Carlos, São Paulo, Brazil. Biota Neotrop.: http//www.biotaneotropica.org.br/v4n2pt/abstract?shortcommunication+bn03604022004

SILVA, W.M. \& MATSUMURA-TUNDISI, T. 2005a. Taxonomy, ecology, and geographical distribution of the species of the genus Thermocyclops kiefer, 1927 (Copepoda, Cyclopoida) in São Paulo State, Brazil, with description of a new species. Braz. J. Biol. 65(3):521-531. http://dx.doi. org/10.1590/S1519-69842005000300018

SILVA, W.M. \& MATSUMURA-TUNDISI, T. 2005b. DNA extraction and ITS2 (internal transcribed spacer 2) gene sequences of some Brazilian freshwater copepods. Verh. Int. Verein. Limnol. 29:409-413.

SILVA, W.M. 2003. Diversidade dos Cyclopoida (Copepoda, Crustacea) de água doce do estado de São Paulo: taxonomia, ecologia e genética. Tese de doutorado, Universidade Federal de São Carlos, São Carlos.

SILVA, W.M. (2008).Diversity and distribution of the free-living freshwater Cyclopoida (Copepoda: Crustacea) in the Neotropics.Braz. J. Biol., 68(4, Suppl.): 1099-1106.

TUNDISI, J.G. 1980. Relatório final do projeto "Tipologia de represas do Estado de São Paulo". (FAPESP - Processo:04-Biológicas 79/0156). v.1,2,3. 